\title{
Synthesis and Analysis of Non-recursive Rejection Filters in the Transient Mode
}

\author{
Dmitrii I. Popov, Sergey M. Smolskiy, Member, IEEE ${ }^{1}$
}

\begin{abstract}
The non-recursive rejection filter (RF), which is improved with the purpose of transient acceleration at arriving of the passive interference edge caused by disturbing reflections from fixed or slow-moving objects, is synthesized by the state-variables method. The structural diagram is offered of the tunable RF in the transient with the purpose of improvement of signal extraction effectiveness from the moving targets on the background of the passive interference edge. The comparative analysis is performed of RF effectiveness for fixed and tunable structure in the transient according to the criterion of the normalized interference suppression coefficient and the improvement coefficient of the signal-to-interference ratio. The essential increase of the signal extraction effectiveness from the moving objects on the background on the interference edge for the wide class of the spectral-correlation characteristics at $\mathbf{R F}$ structure modification.
\end{abstract}

Index Terms - effectiveness analysis, clutter edge, variable states, structure adjustment, transient mode, rejection filters

\section{INTRODUCTION}

Radar systems (RS) found an application for solution of a wide circle of problems of civil and military character [1]. Modern multi-functional RSs allow detection and coordinate localization of various objects. The presence of the strong passive (correlated) interference from the fixed or slowly moved objects caused by disturbing reflections (so-called, a clutter) essentially disturbs the normal RS operation leading to receiver front-end overloading as well as signal masking and eventually to the moving target signal disappearing $[2,3]$. The moving target signal detection on the background of passive interference is the one of relevant and complex tasks of received data processing, which is solved in RS of various application [1]. Methods of protection approaches against clutter and coordinate measurement for different types of radars and the used probing signal are described in a series of publications, in particular, in [1-5].

The clutter protection problem is most effectively solved in so-called pulse-Doppler RSs with the small of-duty factor of the probing signal or in RSs with quasi-continuous wave, in which the coherent pulses with high repetition frequency (up to several tens and even hundreds of kilohertz) are used [1,2]. At that, the unambiguous measurement of the Doppler target velocity with high resolution and accuracy are achieved. However, the range measurement is ambiguous and to avoid

Manuscript submitted on 16 April, 2018

${ }^{1}$ Dmitrii I. Popov is with Ryazan State Radio Engineering University, Russia (e-mail: adop@mail.ru).

Sergey M. Smolskiy is with Moscow Power Engineering Institute (National Research University), Russia (e-mail: SmolskiySM@,mail.ru). this, the specific methods must be applied which complicate the signal processing $[1,5]$.

The unambiguous range measurement is achieved in coherent-pulse RSs at high duty-off factor of probing signals, which leads to wide application of such RSs in practice [1]. However, the low pulse repetition frequency does not provide the unambiguous Doppler frequency measurement for the relevant velocities of the wide class of radar objects [3, 4$]$.

The low pulse repetition frequency chosen from the condition of unambiguous range measurement leads to the close location of comb spectral components, which complicates the moving object signal selection on the background of interference that exceeds in power. In this case, the main operation of received data processing is the rejection of interference spectral components. The rejection filter (RF) becomes the main unit of the appropriate processing system.

A problem of moving target selection on the clutter background, properties of the clutter, novel promising methods of the clutter rejection effectiveness growth and various aspects of this problems are permanently described in Russian and foreign scientific-engineering periodic journals [6-16]. Nevertheless, this scientific direction cannot be considered as fully studied. In this paper, we consider improvement of characteristics of rejection filters of the nonrecursive type, which are widely used in the systems of echoes extractions from moving targets on the background of the intensive clutter [1-3]. When the clutter edge arrives to the input of such a RF, the complicated transient mode is observed at its output as far as this clutter is occupied all delay stages of the RF. In this case, non-compensated clutter residues produce a strong background, which masks the desired signal and leads to false alarms of a radar. To struggle against this situation at discrete scanning of the antenna beam we can use the strobbing (gating) of the RF output samples excluding thus the transient mode at the cost of appropriate number lack of the processed samples. In the mode of continuous scanning, in order to exclude the transient mode, is necessary to undertake the addition measures to determine the clutter edge position. Regardless of the scanning mode, reduction of the processed sequence at its restricted duration, in the case of the high coverage rate, is related to effectiveness losses of signal processing.

In above-cited books [1-5] and papers in the periodic editions [6-15], the problem of improvement of rejection filter characteristic with the purpose of transient reduction is not solved. The exclusion is the publication [16], in which the problem of transient acceleration is solved for the recursive rejection filters. This is achieved by means of switching of 
recursive connections after achievement the steady-state mode in the non-recursive part of RF. At that, the structure of the non-recursive part is classical without acceleration of its transients.

In this connection, the improvement of the RF structure with the aim of its transients speeding-up and effectiveness improving of signal extraction on the background of clutter edge is the relevant research task. Exactly this task is considered in the present paper.

\section{THE SYNTHESIS OF IMPROVED REJECTION FILTER}

For the synthesis of the modernized RF structure in the transient mode, similar to [16], we use the method of state variables, which gives the adequate filter description in the time domain. Being a discrete system, the digital nonrecursive RF of $m$-order in the $k$-th time moment can be described by some state vector $\mathbf{X}(k)=\left[x_{n}(k)\right]$, where $x_{n}(k)$ is the state variable corresponding to the output value of the $n$-th delay unit of the RF, $n=\overline{1, m}$. The difference matrix equation of the RF state has a view in the standard form [17]

$$
\mathbf{X}(k+1)=\mathbf{A} \mathbf{X}(k)+\mathbf{B} u(k),
$$

where $\mathbf{A}$ is the matrix of $m \times m$ dimension, which defines the connection between states in $k$-th and $(k+1)$-th time moments, B is a column vector of $m$ dimension describing the dependence between state variables and the input impact $u(k)$.

The difference equation (1) solution, at known processed sample beginning, similar to [16], is the following vector

$$
\mathbf{X}(k)=\mathbf{A}^{k} \mathbf{X}(0)+\sum_{l=0}^{k-1} \mathbf{A}^{k-1-l} \mathbf{B} u(l),
$$

depending on filter parameters and the vector initial state $\mathbf{X}(0)$.

Modernization of the non-recursive RF structure with the aim of transient speeding-up assumes the formation of the initial state vector. A criterion of transient speeding-up is based on the condition of RF output constancy and hence, its state from the moments of clutter arrival

$$
\begin{aligned}
& \mathbf{X}(k+1)=\mathbf{X}(k)=\mathbf{X}(0) \\
& \text { or } \mathbf{X}(k+1)-\mathbf{X}(k)=0 \text { for } k \geq 0 .
\end{aligned}
$$

The squareness of the pulse train envelope, which takes place at discrete observation radar mode and in combination with known time of sample arrival, opens new possibilities of RF transient speeding-up and is the necessary condition of criterion (3) fulfillment. If the envelope shape differs from rectangular one, for example, at continuous scanning, then we must fix the moment of clutter arrival and perform the preliminary sample weighting to provide the envelope squareness.

Actual clutter samples represent a random process with definite fluctuation character, and we may imply the envelope squareness within the limits of the mean value of samples' sequence. On this account, we shall approximate a step at the clutter edge by the stepped input impact with constant amplitude. Then, a limitation superposable to the input sample takes a form

$$
\begin{aligned}
& u(k+1)=u(k)=u(0) \\
& \text { or } u(k+1)-u(k)=0 \text { for } k \geq 0 .
\end{aligned}
$$

The criterion (3) with relations (2) and (4) account leads to equation

$$
\mathbf{A}^{k}(\mathbf{A}-\mathbf{I}) \mathbf{X}(0)+\mathbf{A}^{k} \mathbf{B} u(0)=0,
$$

which solution is the vector of initial state

$$
\mathbf{X}(0)=(\mathbf{I}-\mathbf{A})^{-1} \mathbf{B} u(0),
$$

where $\mathbf{I}$ is the identity matrix.

Thus, a constancy of states and the RF output is provided upon condition that the initial state vector $\mathbf{X}(0)$ takes a value at the moment of clutter arrival, which is proportional, according to (5), to the first clutter sample.

Let us illustrate a synthesis of the improved RF in the transient mode on an example of non-recursive filter of the $3^{\text {rd }}$ order $(m=3)$ for which

$$
\mathbf{A}=\left[\begin{array}{lll}
0 & 1 & 0 \\
0 & 0 & 1 \\
0 & 0 & 0
\end{array}\right], \quad \mathbf{B}=\left[\begin{array}{l}
0 \\
0 \\
1
\end{array}\right] .
$$

In accordance to (5), we obtain

$$
\begin{aligned}
& \mathbf{X}(0)=\left[\begin{array}{rrr}
1 & -1 & 0 \\
0 & 1 & -1 \\
0 & 0 & 1
\end{array}\right]^{-1}\left[\begin{array}{l}
0 \\
0 \\
1
\end{array}\right] u(0)= \\
& =\left[\begin{array}{lll}
1 & 1 & 1 \\
0 & 1 & 1 \\
0 & 0 & 1
\end{array}\right]\left[\begin{array}{l}
0 \\
0 \\
1
\end{array}\right] u(0)=\left[\begin{array}{l}
1 \\
1 \\
1
\end{array}\right] u(0)=\left[\begin{array}{l}
u(0) \\
u(0) \\
u(0)
\end{array}\right] .
\end{aligned}
$$

The value of initial state vector $\mathbf{X}(0)$, which is obtained as a results of a synthesis, in contrast to [16] assumes the sample $u(0)$ arrival at outputs of all RF delay units at the moment of clutter edge appearance. Since in the classic RF diagram, the $u(0)$ sample does not pass to outputs of delay units and, accordingly, the vector $\mathbf{X}(0)=0$, then, to satisfy the condition (6), we must require introduction of units, which perform the RF structure adjustment.

\section{THE STRUCTURAL DIAGRAM OF THE IMPROVED RF}

The structural diagram of the adjustable RF represented in Fig.1 contains the clutter detector $\mathrm{CD}$, the synchronous generator $\mathrm{SG}$, the switch $\mathrm{SW}$, storage devices $\mathrm{SD}_{T}$ performing functions of delay units for samples processed over the period $T$ of its repetition, adders $(\Sigma)$ and weighting units $g(l)$, $l=\overline{0, m}$ [18]. The clutter detector (CD) contains the comparator performing the comparison of the received interference with the specified values, and logical elements for formation of the unitary single [18]. At coincidence of the antenna beam position during discrete scanning and the clutter 


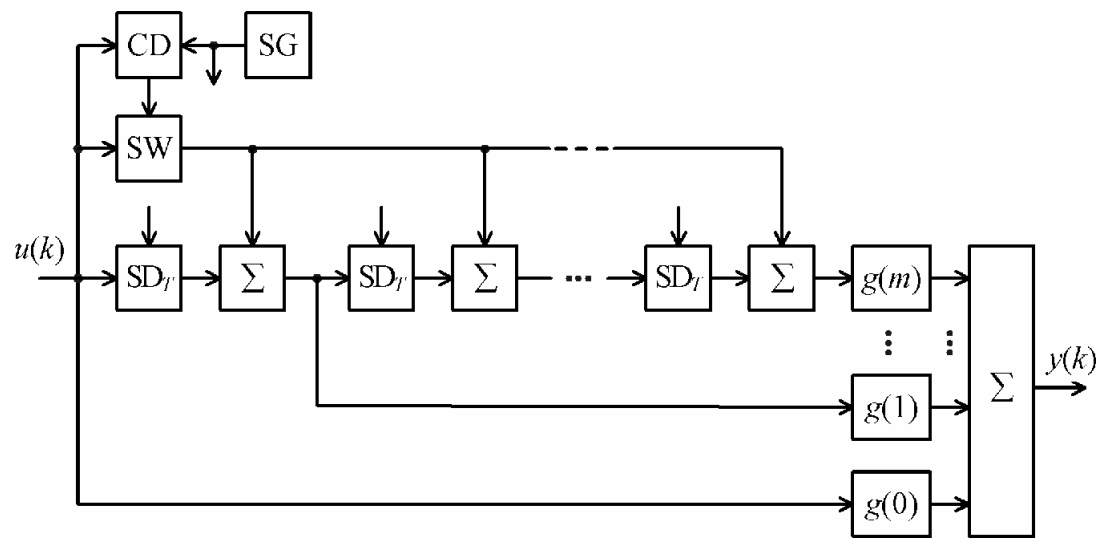

Fig.1. Structural diagram of the adjustable RF

"cloud" edge, the clutter detector CD produces the single voltage signal, and the switch SW, which was earlier in open state, is closed now. The digital sample of the first reflected pulse passes to the first $\mathrm{SD}_{T}$ input and, simultaneously, through the switch SW and adders, to inputs of subsequent $\mathrm{SD}_{T}$. At that, the compensation of clutter component takes place. To the moment of the second reflected pulse arrival, the clutter detector CD opens the switch SW until reflections from the other clutter source begin to appear.

The further processing of samples of the signal and clutter mixture is fulfilled in conventional manner: samples arrive in sequence to the storage devices $\mathrm{SD}_{T}$, weighting units $g(l)$ and the output adder. Samples of the signal mixture and decorrelated (for the received number of periods) interference remainders. Beginning from the first clutter sample, the compensation effectiveness for low-frequency components during the transient mode constantly grows. Echoes from moving targets, which differ from the narrowband clutter by the Doppler modulation, are not compensated and beginning from the second sample pass to RF output. As a whole, effectiveness of Doppler signal extraction on the clutter background in the transient mode increases sequentially from one pulse to the next achieving. After $(m+1)$-th clutter sample arrival, the steady-state value, which corresponds to maximal effectiveness for RF chosen order and parameters.

Synchronous information timing in the storage devices $\mathrm{SD}_{T}$ and the other units provides by the pulses of the synchronous generator $\mathrm{SG}$, which follow with a period of time discretization of the input data. The specific realization of the clutter detector $\mathrm{CD}$ and description of its operation are given in [18].

At continuous observation mode, perfection of the RF structure with the aim of transient speeding-up has its own peculiarities caused by clutter pulse modulation at its edges by the antenna pattern. Detection of the front clutter edge is performed over all modulated pulses of edges till the moments of pulse arrival, which corresponds to the flat plateau of the clutter envelope [19]. Further, the delayed clutter edge samples are weighted with the aim of its squareness recovering, which allows effective extraction of Doppler signals at similar to Fig.1 processing, without waiting of pulses arrival for the envelope flat part. As well, at arrival and detection of the rear clutter edge by means of sample weighting, the recovering of its envelope squareness takes place. After the last pulse arrival, connection between storage devices breaks, which leads to cancellation of the information contained and thus to elimination of the RF transient mode (chink) from the clutter rear edge. Implementation and operation principle of edges detector for continuous observation are described in [19].

The problem of transient speeding-up in the adaptive RF can be solved in the same manner. This is achieved by addition of conventional adaptation units, which perform estimation of spectral-correlation clutter parameters and adjustment of RF characteristics. For this, we may introduce the similar to above-mentioned the detector of clutter edges and appropriate units, which eliminate transients from front and rear edges of the clutter [20].

\section{RF EFFECTIVENESS ANALYSIS IN THE TRANSIENT MODE}

The analysis of RF effectiveness with adjustable structure (Fig. 1) in the transient mode, we perform in the similar manner using the method of state variables. The RF output, as it follows from the matrix equation of input-state-output type, equals

$$
y(k)=\mathbf{C X}(k)+d u(k)
$$

where $\mathbf{C}$ is the vector-line of $m$-dimension describing a connection between RF state and the output value; $d$ is a scalar characterizing a connection between input and output.

Taking (2) into consideration, equation (7) for RF output takes a form

$$
y(k)=\mathbf{C A} \mathbf{A}^{k} \mathbf{X}(0)+\mathbf{C} \sum_{l=0}^{k-1} \mathbf{A}^{k-1-l} \mathbf{B} u(l)+d u(k) .
$$

For RF with fixed structure, the vector $\mathbf{X}(0) \equiv 0$ and expression (8) is converted to the conventional form, which is a convolution of input impact and the weighting function $h(l)$ (pulse response) of RF

$$
y(k)=\sum_{l=0}^{k-1} h(k-l) u(l)+d u(k)=\sum_{l=0}^{k} h(k-l) u(l),
$$

where $h(k-l)=\mathbf{C A}^{k-1-l} \mathbf{B}$ are coefficients of the RF pulse response coinciding with RF weighting coefficients, i.e., $h(k-l)=g(k-l)$.

For RF with adjustable structure, taking into account (5), 
(8), and (9), we have

$$
\begin{aligned}
& y(k)=\mathbf{C A}^{k}(\mathbf{I}-\mathbf{A})^{-1} \mathbf{B} u(0)+\sum_{l=0}^{k-1} h(k-l) u(l)= \\
& =q(k) u(0)+\sum_{l=0}^{k-1} h(k-l) u(l),
\end{aligned}
$$

where $q(k)=\mathbf{C A}^{k}(\mathbf{I}-\mathbf{A})^{-1} \mathbf{B}$.

The term $q(k) u(0)$ defines a contribution to output value formation in the $k$-th time moment at the cost of RF structure adjustment, at that, $q(k)=0$ for $k \geq m$.

The expression (10) can be written as a convolution (9), where coefficients $h(k-l)$ are determined according to the rule

$$
h(k-l)= \begin{cases}g(k-l) & \text { for } l>0, \\ g(k-l)+q(k) & \text { for } l=0 .\end{cases}
$$

The RF effectiveness can be characterized by the normalized (relative the proper receiver noise) clutter suppression coefficient

$$
\begin{aligned}
& \gamma_{\mathrm{cl}}=\left(\sigma^{2} / \sigma_{\mathrm{RF}}^{2}\right)_{\mathrm{cl}} /\left(\sigma^{2} / \sigma_{\mathrm{RF}}^{2}\right)_{\mathrm{n}}= \\
& =\left(\sigma_{\mathrm{RF}}^{2} / \sigma^{2}\right)_{\mathrm{n}} /\left(\sigma_{\mathrm{RF}}^{2} / \sigma^{2}\right)_{\mathrm{cl}},
\end{aligned}
$$

where $\sigma^{2}$ and $\sigma_{\mathrm{RF}}^{2}$ are variances of the clutter or noise, accordingly, at RF input and output.

At zero mean value of initial processes (clutter and noise), the variance at the RF output relative to the $(k+1)$-th pulse of processed sequence is determined in the form $\sigma_{\mathrm{RF}}^{2}=\overline{y^{2}(k)}$. As a result of statistical averaging, taking into account the formula (9) and correlation properties of the clutter and noise, we obtain

$$
\gamma_{\mathrm{cl}}=\sum_{j=0}^{k} h^{2}(j) / \sum_{j, l=0}^{k} h(k-j) h(k-l) \rho(j, l),
$$

where $\rho(j, l)$ are coefficients of the inter-period correlation of the clutter.

Going over to the matrix form, we have

$$
\gamma_{\mathrm{cl}}=\mathbf{H}^{\mathrm{T}} \mathbf{H} / \mathbf{H}^{\mathrm{T}} \mathbf{R}_{\mathrm{cl}} \mathbf{H},
$$

where $\mathbf{H}=[h(k-j)]$ is the $K$-dimension column vector of the RF weighting function, which elements are determined depending on the RF structure (fixed or adjustable); $K=k+1$ is a number of pulses processed in RF; the index "T" means the transposition operation; $\mathbf{R}_{\mathrm{cl}}=[\rho(j, l)]$ is the clutter correlation matrix with $K \times K$ dimension.

As clutter spectral characteristics, we choose boundary approximations for a wide class of the clutter power spectra, which correspond to fast and slow spectrum falling and describing by Gaussian and resonant curves:

$$
\begin{aligned}
& G_{\mathrm{G}}(f)=\exp \left\{-2,8\left[\left(f-f_{0}\right) / \Delta f\right]^{2}\right\}, \\
& G_{\text {res }}(f)=\left\{1+\left[2\left(f-f_{0}\right) / \Delta f\right]^{2}\right\}^{-1},
\end{aligned}
$$

where $f_{0}$ is the central frequency, $\Delta f$ is a spectrum width on a half-level.

Gaussian and exponential correlation functions correspond to these approximations, and coefficients of inter-period correlation have a view

$$
\begin{aligned}
& \rho_{\mathrm{G}}(j, l)=\rho_{\mathrm{G}}^{(j-l)^{2}}, \text { where } \rho_{\mathrm{G}}=\exp \left[-\pi^{2}(\Delta f T)^{2} / 2,8\right] ; \\
& \rho_{\exp }(j, l)=\rho_{\exp }^{|j-l|}, \text { where } \rho_{\exp }=\exp (-\pi \Delta f T) .
\end{aligned}
$$

\section{ANALySis RESUlts}

Let us fulfill the RF effectiveness analysis in the transient operation mode for $K=2,3$, and 4 on the example of nonrecursive $\mathrm{RF}$ the $3^{\text {rd }}$ order with binomial weighting coefficients $g(0)=-g(3)=1, g(1)=-g(2)=-3$.

Values of processing vector $\mathbf{H}$ for different $K$ and for both types of RF: fixed structure (FS) and adjustable structure (AS) are shown in Table 1. We see that RF structure adjustment leads to such values of pulse response coefficients, which correspond to compensation of constant amplitude samples that corresponds to sample decorrelation of the actual clutter at each step of the transient process.

Table 1. Values of the processing vector $\mathbf{H}$

\begin{tabular}{|c|c|c|c|c|}
\hline \multicolumn{1}{|c|}{$\begin{array}{c}\text { K } \\
\text { RF type }\end{array}$} & 1 & 3 & 4 \\
\hline FS & {$[1]$} & {$\left[\begin{array}{r}-3 \\
1\end{array}\right]$} & {$\left[\begin{array}{r}3 \\
-3 \\
1\end{array}\right]$} & {$\left[\begin{array}{r}-1 \\
3 \\
-3 \\
1\end{array}\right]$} \\
\hline AS & {$[0]$} & {$\left[\begin{array}{r}-1 \\
1\end{array}\right]$} & {$\left[\begin{array}{r}2 \\
-3 \\
1\end{array}\right]$} & {$\left[\begin{array}{r}-1 \\
3 \\
-3 \\
1\end{array}\right]$} \\
\hline
\end{tabular}

The specific expressions for $\gamma_{\mathrm{cl}}$ coefficient for both approximations of the clutter correlation functions, which are obtained by formula (11), are shown in Table 2.

Functions $\gamma_{\mathrm{cl}}(K)$ for the RF fixed structure (dashed line) and for the RF adjustable structure (solid lines) calculated according to Table 2 expressions for $\rho_{\mathrm{G}}=\rho_{\exp }=0,99$ are shown in Fig. 2. Characteristics with number 1 correspond to the Gaussian function of clutter correlation; characteristic with number 2 correspond to the exponential function. As we see, for adjustable structure of RF, the significant growth of clutter suppression effectiveness can be achieved. 
Table 2. Specific expressions for $\gamma_{c l}$ coefficient

\begin{tabular}{|c|c|c|c|}
\hline$K$ & RF type & Gaussian correlation function & Exponential correlation function \\
\hline \multirow{2}{*}{2} & FS & $10 /\left[4+6\left(1-\rho_{\mathrm{G}}\right)\right]$ & $10 /\left[4+6\left(1-\rho_{\exp }\right)\right]$ \\
\cline { 2 - 4 } & AS & $1 /\left(1-\rho_{\mathrm{G}}\right)$ & $1 /\left(1-\rho_{\exp }\right)$ \\
\hline \multirow{2}{*}{3} & FS & $19 /\left[19\left(1-\rho_{\mathrm{G}}\right)-5 \rho_{\mathrm{G}}\left(1-\rho_{\mathrm{G}}^{3}\right)+\rho_{\mathrm{G}}^{4}\right]$ & $19 /\left[\left(1-\rho_{\exp }\right)\left(19-5 \rho_{\exp }\right)+\rho_{\exp }^{2}\right]$ \\
\cline { 2 - 4 } & AS & $14 /\left[14\left(1-\rho_{\mathrm{G}}\right)-4 \rho_{\mathrm{G}}\left(1-\rho_{\mathrm{G}}^{3}\right)\right]$ & $14 /\left(1-\rho_{\exp }\right)\left(14-4 \rho_{\exp }\right)$ \\
\hline 4 & FS and AS & $20 /\left[20\left(1-\rho_{\mathrm{G}}\right)-10 \rho_{\mathrm{G}}\left(1-\rho_{\mathrm{G}}^{3}\right)+2 \rho_{\mathrm{G}}^{4}\left(1-\rho_{\mathrm{G}}^{5}\right)\right]$ & $20 /\left(1-\rho_{\exp }\right)\left(20-10 \rho_{\exp }+2 \rho_{\exp }^{2}\right)$ \\
\hline
\end{tabular}

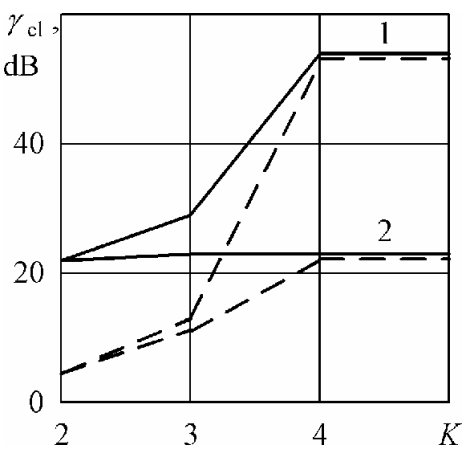

Fig. 2. Functions of the clutter suppression coefficient

For $K=2$ the benefit in $\gamma_{\mathrm{cl}}$ coefficient (compared to RF without adjustment) is $17 \mathrm{~dB}$, for $K=3-16 \mathrm{~dB}$ for the Gaussian function and $12 \mathrm{~dB}$ for the exponential function. If $K=4$, the steady-state mode of RF operation occurs. The suppression effectiveness of the clutter with fast spectrum decrease is shown essentially higher than with slow decrease. However, in the last case, during RF structure the steady-state mode of clutter suppression is achieved practically right away.

Let us consider the signal extraction effectiveness in the $\mathrm{RF}$, which can be characterized by the improving coefficient of a signal/clutter ratio

$$
\mu=\mathbf{H}^{\mathrm{T}} \mathbf{R}_{\mathrm{s}} \mathbf{H} / \mathbf{H}^{\mathrm{T}} \mathbf{R}_{\mathrm{cl}} \mathbf{H},
$$

where $\mathbf{R}_{\mathrm{s}}=\left[\rho_{\mathrm{s}}(j, l)\right]$ is the correlation matrix of the $\mathrm{RF}$ input signal, $\mathbf{H}^{\mathrm{T}} \mathbf{R}_{\mathrm{s}} \mathbf{H}=\gamma_{\mathrm{s}}$ is the signal transmission factor through RF.

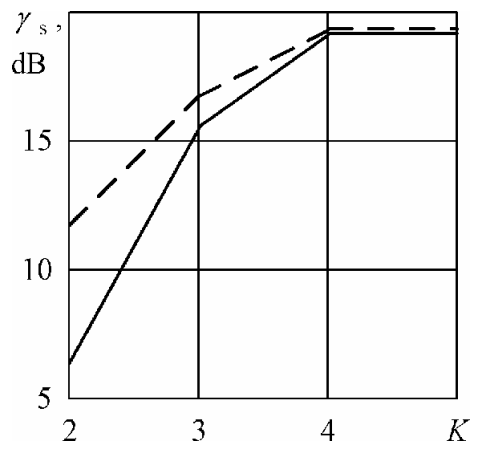

Fig. 3. Functions of signal transmission factor
Functions of $\gamma_{\mathrm{s}}(K)$ and $\mu(K)$, respectively, are shown in Figs. 3 and 4 for the optimal target velocity, mutual signal fluctuations $\left[\rho_{\mathrm{s}}(j, l)=(-1)^{j+l}\right]$ and former clutter characteristics (designations correspond to Fig. 2). It follows from Fig. 3 that under RF structure adjustment and $K=2$ and 3 , the rather small loss in signal transmission through the RF takes place, which can be explained by differences in $\mathbf{H}$ vector elements for RF of both types (see Table 1). Resulting effectiveness of signal extraction on the background of clutter edge, as we see from Fig. 4, for the filter with adjustable

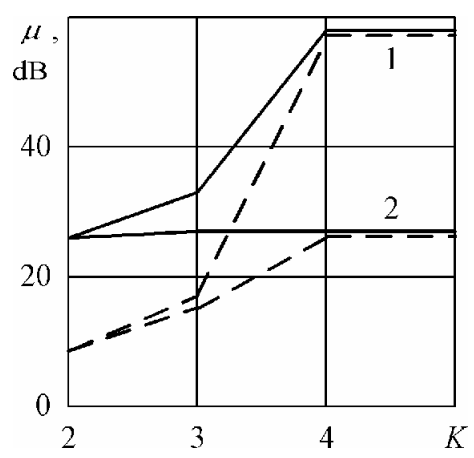

Fig. 4. Functions of improvement factor for signal/clutter

structure, is higher than effectiveness of conventional RF with the fixed structure by $12-17 \mathrm{~dB}$.

\section{CONCLUSION}

The improved non-recursive RF synthesized in this paper using the method of state variables allows performing speeding-up of the transient process by means of setting the initial RF state, which is proportional to the first clutter sample at the moment of its arrival.

RF modernization is achieved by its structure adjustment according to results of clutter edge detection, which leads to its effectiveness increase in the transient mode sequentially from one pulse to another.

The comparative RF analysis for fixed and adjustable structures performed by authors on the base of the state variables method shows that adjustment of RF structure ensures significant effectiveness growth in the transient mode for moving targets on the background of clutter edges for a wide class of spectral-correlation characteristics. 


\section{REFERENCES}

[1] Radar Handbook / Ed. by M. I. Skolnik. - 3rd ed. - McGraw-Hill, 2008. - $1352 \mathrm{p}$

[2] Melvin, W.L., Scheer, J.A. (Eds.). Principles of Modern Radar: Advanced Techniques. - New York: SciTech Publishing, IET, Edison, 2013. - 846 p.

[3] Richards, M.A. Fundamentals of Radar Signal Processing, Second Edition. - New York: McGraw-Hill Education, 2014. - 618 p.

[4] Digital signal processing in multi-functional radars. Methods. Algorithms. Equipment / under edition of G.V. Zaitsev (in Russian). Moscow: Radiotekhnika Publ., 2015. - 376 p.

[5] Lozovskiy, I.F. Digital signal processing in surveillance radars (in Russian). - Novosibirsk: NSTU Publ., 2016. - 270 p.

[6] Cui, Y., Yamaguchi, Y., Kobayashi, H. Yang, J., Singh, G., Park, S.-E. On Semiparametric Clutter Estimation for Ship Detection in Synthetic Aperture Radar Images // IEEE Transactions on Geoscience and Remote Sensing. - 2013. - Vol. 51. - No 5. - P. 3170-3180. DOI: $10.1109 /$ TGRS.2012.2218659.

[7] Bezruk, V.M., Tykhonov, V.A., Kudriavtseva, N.V. Clutter Suppression Using Additive Linear Prediction Filters // Telecommunications and Radio Engineering. - 2013. - Vol. 72. - No 9. - P. 819-828. DOI: 10.1615/TelecomRadEng.v72.i9.70.

[8] Zhang, L.-Z., Lu, B.-Y., Zhou, Z.-M., Sun, X. The Clutter Suppression Based on Factor Analysis and Image Contrast in Through-the-wal Application // Dianzi Yu Xinxi Xuebao. - 2013 . - T. 35. - No 11. - P. 2686-2692. DOI: 10.3724/SP.J.1146.2013.00063

[9] Rodionov V.V. Noise-immunity of adaptive pulse-Doppler detectors on the clutter background (in Russian). // Antennas. - 2014. - No 1 (200). - P. 023-029.

[10] Gordeev A.Yu., Yatsyshen V.V. Promising methods of effectiveness increase for clutter suppression by systems of moving target selection. (in Russian). // Electromagnetic waves and electronic systems. - 2015. - Vol. 20. - No 3. - P. 40-52.

[11] Kutepov V.E. Doppler filtering of clutter and disturbing reflections on the super-scale range (in Russian). // Uspekhi covremennoi radioelectroniki. - 2016. - No 2. - P. 104-107.

[12] Gracheva, V., Ender, J. Multichannel Analysis and Suppression of Sea Clutter for Airborne Microwave Radar Systems // IEEE Transactions on Geoscience and Remote Sensing. - 2016. - Vol. 54. - No 4. - P. 2385-2399.

DOI: 10.1109/TGRS.2015.2500918

[13] Dmitrii I. Popov and Sergey M. Smolskiy, "Estimation of the Clutter Correlation Coefficient in Radar Systems", Infocommunications Journal, Vol. VIII, No 3, September 2016, pp. 8-12.

[14] Dmitrii I. Popov and Sergey M. Smolskiy, "Optimization of the digital rejection filter", Infocommunications Journal, Vol. IX, No 2, June 2017, pp. 1-5.

[15] Ananenkov A.E., Marin D.V., Nuzhdin V.M., Rastorguev V.V. Estimation of clutter suppression level in radar for detection of smallsize flying vehicles (in Russian). // Electrosviaz. - 2017. - No 11. - P. 54-57.

[16] Popov, D.I., Smolskiy, S.M. Signal processing in recursive rejection filters in the transient mode // Turkish Journal of Electrical Engineering \& Computer Sciences, 26, No 1, 2018,pp. 194-203 DOI: 10.3906/elk-1705-215.

[17] Director, S.W.; Rohrer, R.A. Introduction to Systems Theory, McGrawHill Book Company, New York, 1972. - 464 p.

[18] Guskov, S.V.; Popov, D.I. Device for interference suppression. SU Patent No. 1083367. MKP H04B 1/10. Byull. Izobret., n. 12, 1984 Claimed 06.01.1982. Published 30.03.1984.

[19] Guskov, S.V.; Ivanov, V.A.; Popov, D.I. Rejection filter. SU Patent No. 1679874. MKP G01S 13/52. Byull. Izobret., n. 23, 1999. Claimed 16.01.1984. Published 20.08.1999.

[20] Popov, D.I.; Gerasimov, S.V.; Mataev, E.N. Device for adaptive suppression of interference. SU Patent No. 1802616. MKP G06F 17/17, H03H 21/00. Byull. Izobret., n. 22, 1996. Claimed 19.04.1990. Published 10.08.1996.

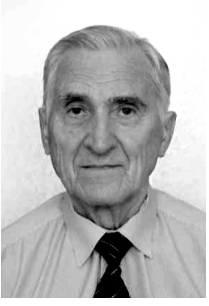

Dmitrii I. POPOV, born in 1939, in Ryazan, Ph.D. in Engineering, Dr. Sc. in Engineering, full professor of Radio Engineering Systems Department in Ryazan State Radio Engineering University (RSREU). Graduated from RSREU in 1961 on specialty "Designing and manufacture of radio equipment". Ph.D. from 1967, Dr. Sc. from 1990.

$\mathrm{PhD}$ thesis in specialty "Radar and Radio Navigation Technologies" on theme "Research of detection systems effectiveness for fluctuating signals on the clutter background". Dr. Sc. thesis in specialty "Radar and Radio Navigation Technologies" on theme "Synthesis and analysis of adaptive systems for moving target selection". The active member of International Academy of Informatization.

Author of about 350 scientific publication and patents. Field of research: theory and technique of radar signals processing against noises.

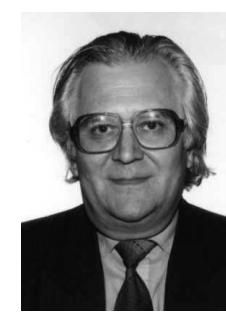

Sergey M. SMOLSKIY, born in 1946, Ph.D. in Engineering, Dr. Sc. in Engineering, full professor of Department of Radio Signals Formation and Processing of the National Research University "MPEI". After graduation of Ph.D. course and defense of $\mathrm{Ph} . \mathrm{D}$. thesis in 1974 he worked in Department of Radio Transmitting Devices of MPEI, where was engaged in theoretical and practical problems of development of transmitting cascades of short-range radar. In 1993 defended the Doctor of Science thesis and now he works as a professor of Radio Signals Formation and Processing Dept. Academic experience - over forty years. The list of scientific works and inventions contains over three hundreds of scientific articles, 15 books, three USSR copyright certificates on invention. The active member of International Academy of Informatization, International Academy of Electrotechnical Sciences, International Academy of Sciences of Higher Educational Institutions. The active member of IEEE. 